\title{
Cultura de seguridad del paciente en Hospitales Regionales de Alta Especialidad
}

\section{Culture of patient safety in High Specialty Regional Hospitals}

\author{
Mario A. Banderas-Ocampo ${ }^{1}$, Elvira A. López-Jacinto ${ }^{2}$, Claudia Pimentel-Roque ${ }^{3}$, Glendy A. \\ Bojórquez-Sabido ${ }^{4}$, Leticia Ramírez-Contreras ${ }^{5}$, Dalila Chan-Molina ${ }^{6}$, Sandra O. Ayala-Casas ${ }^{7}$, \\ José L. Palafox-García ${ }^{7}$, Elizabeth Llanas-Olmos ${ }^{8}$ y Diana L. Velázquez-Castañeda ${ }^{*}$ \\ ${ }^{1}$ Comisión Coordinadora de Institutos Nacionales de Salud y Hospitales de Alta Especialidad, Ciudad de México; ${ }^{2}$ Gestión de Calidad,, Hospital Regional de Alta \\ Especialidad de Ixtapaluca, Edo. Mex.; ${ }^{3}$ Gestión de Calidad, Hospital Regional de Alta Especialidad de Oaxaca, San Bartolo Coyotepec, Oax.; 4 Gestión de Calidad, \\ Hospital Regional de Alta Especialidad de la Península de Yucatán, Mérida, Yuc.; ${ }^{5}$ Gestión de Calidad, Hospital de Especialidades Pediátricas, Tuxtla Gutiérrez,Chis.; \\ ${ }^{6}$ Gestión de Calidad, Hospital Regional de Alta Especialidad Ciudad Salud, Tapachula, Chis.; ' Gestión de Calidad, Hospital Regional de Alta Especialidad del Bajío, \\ León, Gto.; ${ }^{8}$ Gestión de Calidad, Hospital Regional de Alta Especialidad de Ciudad Victoria, Tamps. México
}

\begin{abstract}
The objective is to describe the perception of medical and nursing staff regarding the degree of culture of patient safety. Method: The questionnaire on Patient Safety Culture in Mexico was applied by means of a survey to employees assigned to clinical areas of the High Specialty Regional Hospitals. Results: The degree of patient safety in the HRAE was recorded at 8.35 (decimal scale); comparatively, the national average in the same year was 7.81. In HRAE, the perceived positive dimensions are: teamwork, hospital support, organizational learning, and reported events. In contrast, the dimensions with negative perception were: personnel assignment, actions of the boss to promote safety, and shift changes and transitions in the hospital. Discussion: The result obtained when compared with those of 2018, allows to conclude that the HRAE continue to improve their processes and consolidate the culture of patient safety, which enables the assigned personnel to develop their daily practices in a safe environment.
\end{abstract}

Key words: Patient safety. Continuous improvement. High Specialty Hospitals.

\section{RESUMEN}

El objetivo es describir la percepción del personal médico y enfermería respecto al grado de cultura de seguridad del paciente. Método: Se aplicó mediante encuesta el cuestionario sobre Cultura de Seguridad del Paciente en México a empleados adscritos en áreas clínicas de los Hospitales Regionales de Alta Especialidad. Resultados: Se registró en 8.35 (escala decimal) el grado de seguridad del paciente en los HRAE; comparativamente, la media nacional en el mismo año fue de 7.81. En los HRAE las dimensiones positivas percibidas son: trabajo en equipo, apoyo del hospital, aprendizaje organizacional y eventos reportados. En contraste, las dimensiones con percepción negativa fueron: asignación de personal, acciones del jefe para promover la seguridad, y cambios de turno y transiciones en el hospital. Discusión: El resultado obtenido al compararse con los del año 2018, permite concluir que los HRAE continúan mejorando sus procesos y consolidando la cultura de seguridad del paciente, lo cual posibilita que el personal adscrito desarrolle sus prácticas cotidianas en un ambiente seguro.

Palabras clave: Seguridad del paciente. Mejoramiento de la calidad. Hospitales Especializados.

\section{Correspondence:}

*Diana L. Velázquez-Castañeda

E-mail: maosdiana@gmail.com
Date of reception: 04-02-2020

Date of acceptance: 07-10-2020

DOI: 10.24875/HMCM.20000004
Available online: $18-11-2020$

Hosp Med Clin Manag. 2020;13:116-23

2604-0018 / @ 2020 Mexican Regional Hospitals of High Specialty and Federal Hospitals. Published by Permanyer. This is an open access article under the CC BY-NC-ND license (http://creativecommons.org/licenses/by-nc-nd/4.0/). 


\section{INTRODUCCIÓN}

«Beneficencia y no maleficencia» es un principio ético de hacer el bien y evitar el mal, de promover el bien, prevenir y no infligir daño o maldad. Junto con la expresión primum non nocere, traducida como «lo primero es no hacer daño", constituyen la base de la cultura de seguridad del paciente (SP) en un establecimiento de salud ${ }^{1}$. Sin embargo, el tránsito del paciente en la atención médica se ve inmerso en diversos riesgos, en los que las barreras de seguridad se omiten o se minimizan, resultando eventos que afectan al paciente.

En México se estima que el $8 \%$ de los pacientes hospitalizados sufre algún tipo de daño y el $2 \%$ muere, siendo la población más afectada la que se encuentra económicamente activa, situación que pone de manifiesto repercusiones sociales que pocas veces nos detenemos a mirar. La ventana de oportunidad se encuentra en los eventos adversos (EA) que son prevenibles y en ellos es donde se debe incidir para brindar atención médica más segura² ${ }^{2}$

\section{Antecedentes}

En el año 2007 México participó en el Estudio de Prevalencia de Efectos Adversos en Hospitales de Latinoamérica (IBEAS, 2007), con una muestra que incluyó 2,897 pacientes de 28 hospitales, encontrando que la prevalencia del EA fue del $7.7 \%^{3}$. Por su parte, el entonces Sistema Integral de Calidad en Salud (SICALIDAD) emitió diversas instrucciones para el despliegue de estrategias encaminadas a sentar las bases en materia de calidad y SP en México. Entre ellas, la instrucción 337, emitida en el año 2012, estableció «llevar a cabo la evaluación de la cultura de seguridad del paciente utilizando el cuestionario adaptado por la Dirección General de Calidad y Educación en Salud (DGCES) ${ }^{4}$.

Posteriormente, en una acción conjunta entre el Consejo de Salubridad General (CSG) y la DGCES, se publicó en el Diario Oficial de la Federación el Acuerdo por el que se declara la obligatoriedad de la implementación, para todos los integrantes del Sistema Nacional de Salud, del documento denominado Acciones Esenciales para la Seguridad del Paciente (AESP), en el que se incluye la AESP 8, Cultura de Seguridad del Paciente ${ }^{2}$.
Bajo este contexto, toda unidad de salud deberá incorporar en el sistema de gestión de calidad estrategias para reducir el riesgo asociado al proceso de atención y con ello dar cumplimiento a las AESP, considerando que la evaluación del nivel de implementación quedará a cargo del CSG por medio del Modelo de Seguridad del Paciente propuesto para la certificación hospitalaria. Por su parte la DGCES en su atribución de rectoría a través de la evaluación de la calidad y mejora de procesos, quienes promueven mediante el Modelo de Gestión de Calidad y el proceso de Acreditación el cumplimiento de dicha normativa.

\section{MEDICIÓN DE LA CULTURA DE SEGURIDAD}

Particularmente, la medición de la cultura de seguridad en el ámbito hospitalario se desarrolló en el 2004 por la Agency for Healthcare Research and Quality (AHRQ), con el cuestionario denominado Hospital survey on patient safety culture. Este fue traducido del inglés al español por un grupo de la Universidad de Murcia, adaptándolo para su aplicación.

En México, la Comisión Nacional de Arbitraje Médico (CONAMED) y posteriormente la DGCES realizaron la adecuación y validación del cuestionario, el cual se aplicó por primera vez en el 2009 como estudio piloto y posteriormente en 12 hospitales del Sistema Nacional de Salud.

En la actualidad la medición se realiza de forma anual y es voluntaria para todo establecimiento de atención médica en los tres niveles de atención. El proceso operativo lo establece la DGCES por medio de los responsables estatales de calidad (REC) y quienes lideran la estrategia de evaluación al interior de cada unidad, al menos en los HRAE, son los gestores de calidad con sus equipos de trabajo, para lograr en tiempo y forma las actividades logísticas para la aplicación del cuestionario. El compromiso de las unidades participantes es presentar ante el Comité de Calidad y Seguridad del Paciente (COCASEP) los resultados y atender las áreas de oportunidad identificadas. Con esta acción sustantiva se muestra que el ciclo de gestión de calidad es vital para la mejora continua e incide directamente en la cultura de SP.

Los resultados trajeron consigo la oportunidad de formular para el presente estudio la siguiente pregunta: ¿Cuál es el grado de cultura de SP en los HRAE? 


\section{MÉTODOS}

El estudio (observacional, descriptivo y transversal) empleó una encuesta como método de recogida de los datos. El instrumento que se utilizó fue el cuestionario con escala tipo Likert emitido por la DGCES. Los reactivos se dividen en 9 secciones, que a su vez pretenden explorar 12 dimensiones que inciden en la SP:

1. Su área de trabajo.

2. Su supervisor/jefe.

\section{Comunicación.}

4. Frecuencia de eventos reportados.

5. Grado de SP.

6. Su hospital.

7. Número de eventos reportados.

8. Información complementaria.

\section{Sus comentarios.}

Además, incluye dos preguntas adicionales: el grado general sobre la SP y el número de incidentes/eventos reportados.

Se consideró como criterio de inclusión: ser personal profesional de la salud adscrito a los siete HRAE, que son: el HRAE de Ciudad Salud (HRAECS), el de Oaxaca (HRAEO), el de Ixtapaluca (HRAEI), el de Ciudad Victoria (HRAECV), el del Bajío (HRAEB) y el Hospital de Especialidades Pediátricas (HEP); realizar funciones en área clínica; aceptar participar, y responder a todos los puntos del cuestionario. Los criterios de exclusión fueron: cuestionarios incompletos, ser personal médico y de enfermería adscrito a áreas administrativas, o bien que no realiza actividades relacionadas con el cuidado directo del paciente.

La encuesta estuvo disponible en línea durante el periodo del 29 de agosto al 13 de septiembre del 2019. Cabe mencionar que la plataforma tiene dos mecanismos para obtener los resultados: el primero, por medio de gráficas y tablas con las frecuencias por dimensión evaluada, y el segundo mediante la descarga de la base de datos con las frecuencias por dimensión.
El análisis consideró la clasificación de resultados establecidos por la $A H R Q^{5}$, en la que una dimensión es considerada como "fortaleza» para el hospital cuando obtiene un valor igual o mayor al $75 \%$ de respuestas. Si este criterio no se cumple, la dimensión es considerada como "oportunidad de mejora».

\section{RESULTADOS}

El tamaño de la muestra por conveniencia fue de 991 trabajadores de la salud, de los cuales 600 son personal de enfermería y 391 personal médico. La distribución de la muestra por HRAE se muestra en la figura 1.

Una de las variables que se exploró fue la antigüedad laboral del personal que participó en la encuesta, considerando que los HRAE tienen entre 6 y 13 años de inicio de operación (Fig. 2).

Con la intención de focalizar las acciones a implementar en los Planes de Mejora de la Cultura de Seguridad del Paciente (PMCSP) de los HRAE se analizaron los resultados de las principales dimensiones negativas que percibe el personal, identificando que las estrategias debían incidir en: la pérdida de información y problemas durante los cambios de turno o transferencia de pacientes entre servicios; la suficiencia, disponibilidad y gestión del personal; y en mejorar la retroinformación, es decir, fortalecer en el jefe o supervisor la capacidad de escucha y grado de interés por la SP (Fig. 3).

No obstante, al analizar el total de las dimensiones evaluadas, en la gráfica de Pareto se observa que la cultura organizacional se debe fortalecer mediante el trabajo en equipo y la comunicación, incluso fomentando mensajes que minimicen la actitud punitiva ante un error, propiciando un clima de confianza en el cual el aprendizaje y la retroalimentación sean elementos permanentes que contribuyan a mejorar la SP (Fig. 4).

Por otra parte, al comparar los resultados del informe emitido por la DGCES (en el que se indica cuál ha sido la media nacional del grado de SP desde el año 2014 hasta los resultados obtenidos en el año 2019) con la media de SP obtenida en las unidades participantes de los tres grandes bloques de hospitales que conforman la Comisión Coordinadora de Institutos Nacionales de Salud y Hospitales de Alta Especialidad (CCINSHAE), 


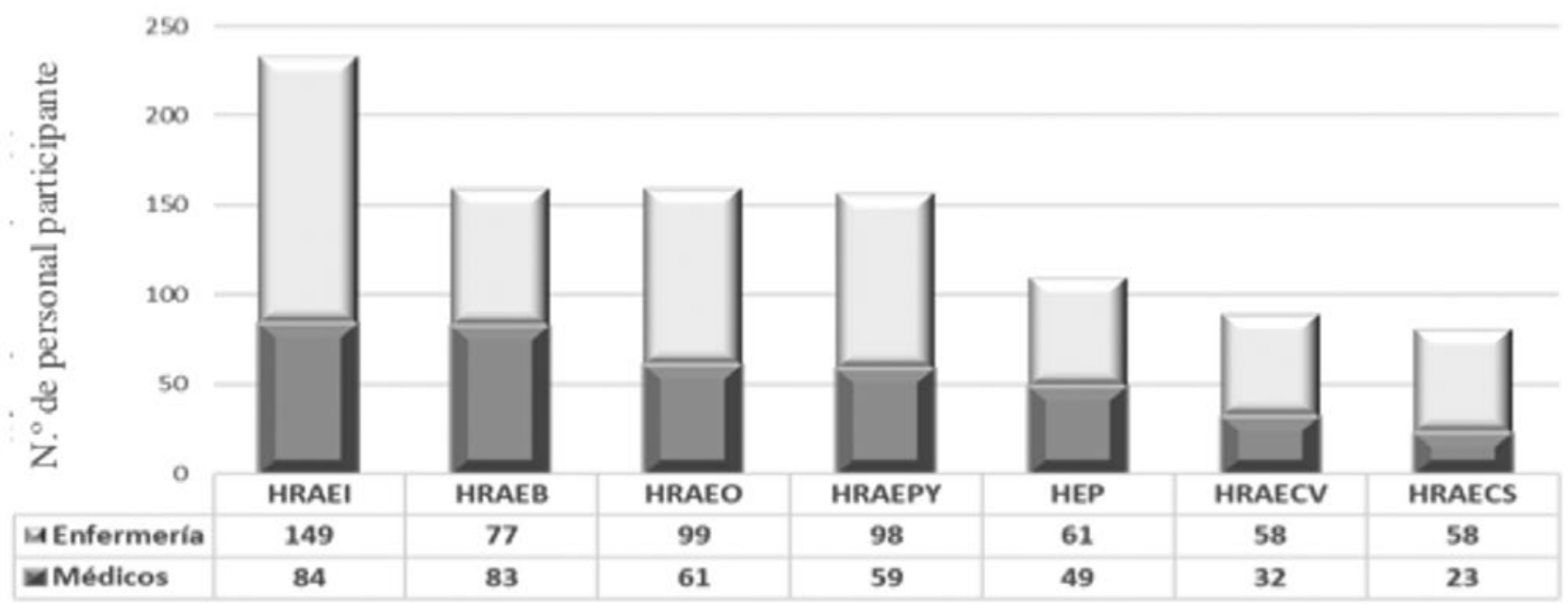

Figura 1. Evaluación de la cultura de seguridad del paciente 2019. Distribución de la muestra en los HRAE *Fuente: elaboración propia a partir de la Encuesta sobre la cultura de seguridad del paciente, 20196. HRAEl: HRAE de Ixtapaluca; HRAEB: HRAE del Bajío; HRAEO: HRAE de Oaxaca; HRAEPY: HRAE de la península de Yucatán; HEP: Hospital de Especialidades Pediátricas; HRAECV: HRAE de Ciudad Victoria; HRAECS: HRAE de Ciudad Salud.

Institutos Nacionales de Salud (INS) los Hospitales Federales de Referencia (HFR) y los HRAE, se observa un comportamiento similar entre ellos, pero además crece la expectativa por continuar implementado acciones que vayan encaminadas a ser congruentes con los resultados obtenidos (Fig. 5).

\section{DISCUSIÓN}

La Clasificación Internacional de Seguridad del Paciente refiere que las organizaciones con una cultura de la seguridad eficaz reconocen que las actividades de su organización son de alto riesgo y propensas a los errores, por lo que propician un entorno no punitivo en el que los individuos puedan notificar los errores sin ser castigados. Son entornos que trabajan de manera multidisciplinaria para encontrar vulnerabilidades y encontrar las mejores soluciones correctivas que se presentan en la SP7. También se ha identificado que son organizaciones que reconocen que existen riesgos en sus actividades, con la expectativa de encontrar la colaboración para las soluciones y en donde se destinan recursos para la SP.

Un aspecto que no puede omitirse es que entre más apertura exista para la notificación no punitiva, mayor aprendizaje se tiene de los errores; en cambio, el

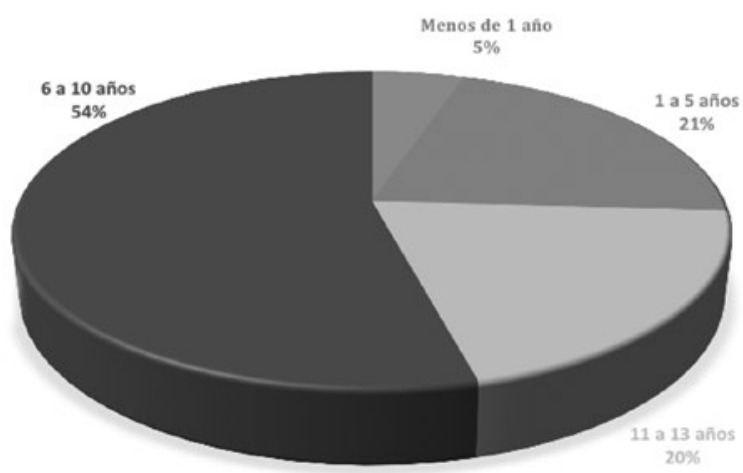

Figura 2. Distribución del personal según años de antigüedad laboral en los HRAE (2019). HRAE: hospitales regionales de alta especialidad.

ocultar los errores por miedo a la sanción genera un ambiente con una cultura de seguridad nula o deficiente.

La encuesta de Cultura de Seguridad del Paciente reveló que los «problemas en cambios de turno y transiciones entre servicios» es la principal dimensión negativa, siendo la falta de comunicación entre los profesionales de la salud un factor que puede aumentar la ocurrencia de EA. 


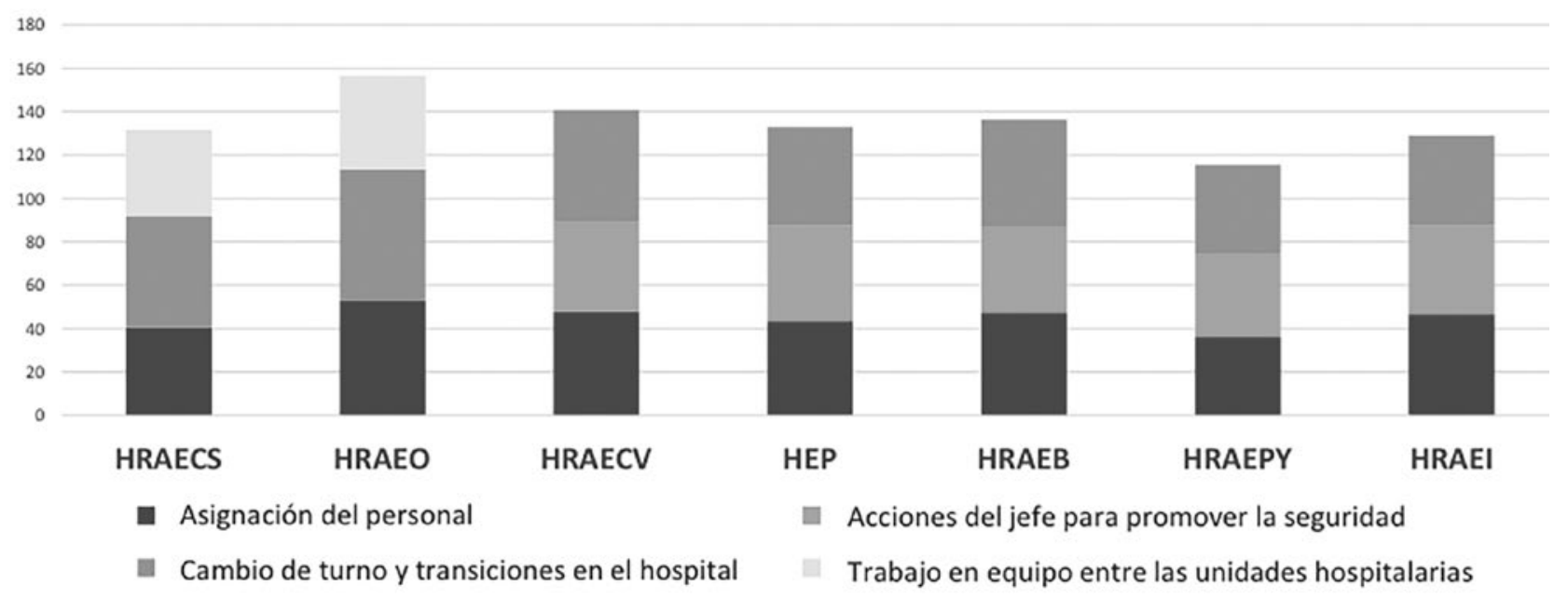

Figura 3. Resultados 2019 de la Encuesta de seguridad del paciente en los hospitales regionales de alta especialidad (HRAE). Principales dimensiones negativas que percibe el personal en los HRAE*. *Fuente: elaboración propia a partir de la encuesta sobre la Cultura de seguridad del paciente, $2019^{6}$. HRAEI: HRAE de Ixtapaluca; HRAEB: HRAE del Bajío; HRAEO: HRAE de Oaxaca; HRAEPY: HRAE de la península de Yucatán; HEP: Hospital de Especialidades Pediátricas; HRAECV: HRAE de Ciudad Victoria; HRAECS: HRAE de Ciudad Salud.

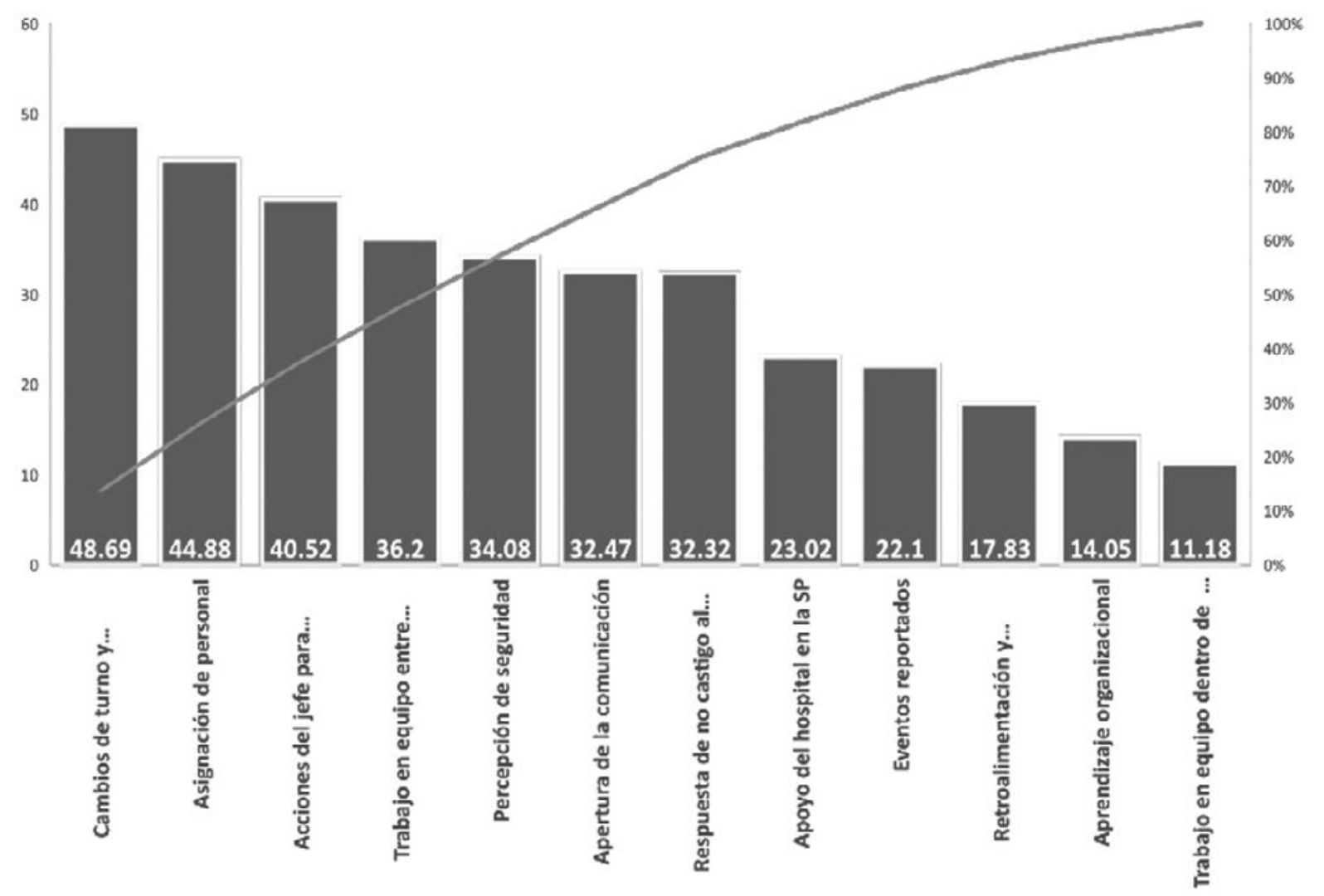

12 dimensiones

Figura 4. Gráfica de Pareto para respuestas negativas por dimensión. Resultados 2019 de la Encuesta de seguridad del paciente, promedio en los hospitales regionales de alta especialidad (HRAE)*. *Fuente: elaboración propia a partir de la encuesta sobre la Cultura de seguridad del paciente, $2019^{6}$. SP: seguridad del paciente. 


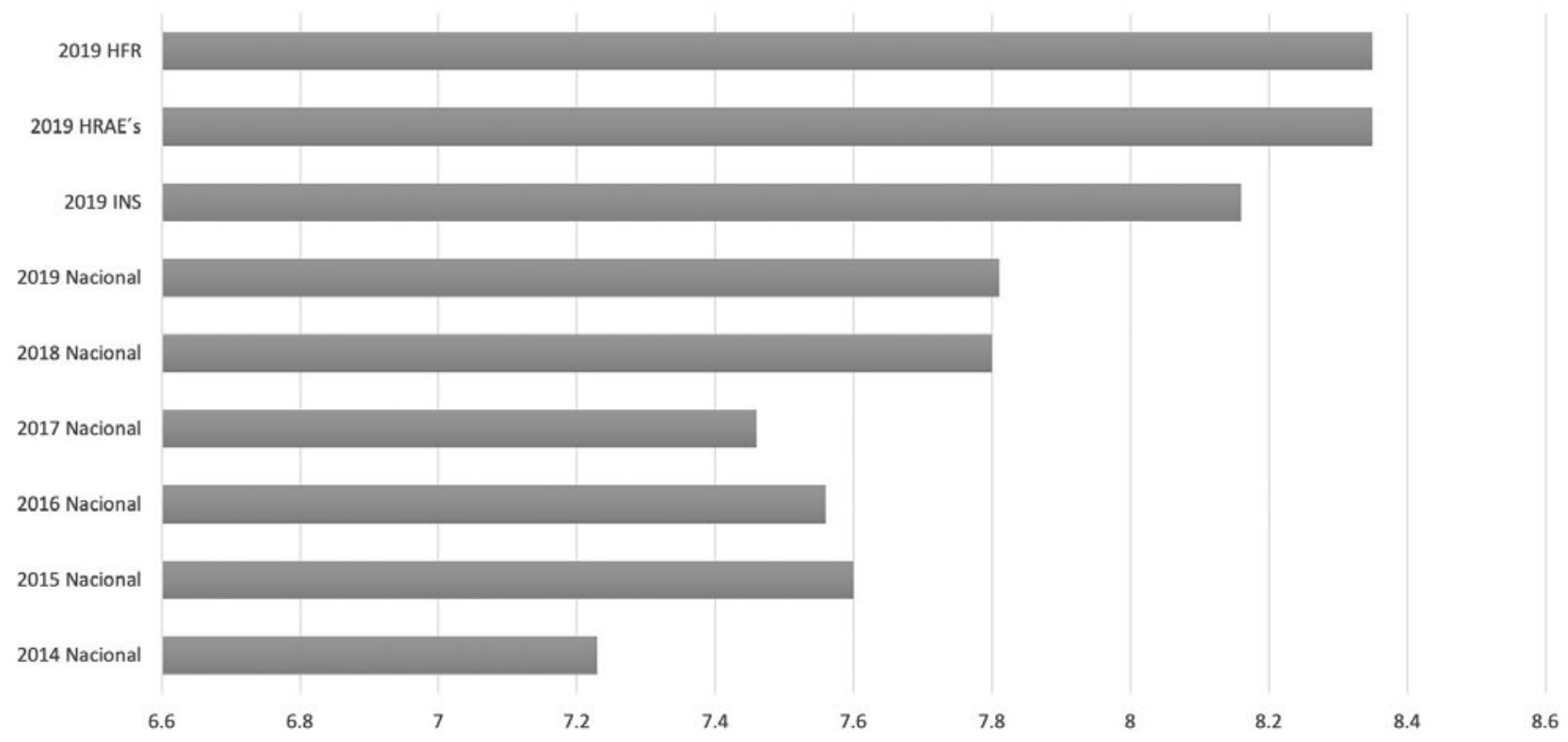

Figura 5. Resultados comparativos 2014-2019 del grado de seguridad del paciente nacional y los resultados en CCINSHAE (INS, HFR y HRAE)*. *Fuente: elaboración propia a partir de la encuesta sobre la Cultura de seguridad del paciente, 20196. CCINSHAE: Comisión Coordinadora de Institutos Nacionales de Salud y Hospitales de Alta Especialidad; INS: Instituto Nacional de Salud; HFR: hospitales federales de referencia; HRAE: hospitales regionales de alta especialidad.

La Organización Mundial de la Salud estima que cada año decenas de millones de pacientes sufren lesiones incapacitantes o mueren como consecuencia de prácticas médicas o atención insegura ${ }^{8}$. Como parte de la concienciación se considera que los errores son producto del sistema. Este nuevo enfoque no deja de lado la conducta y responsabilidad de cada persona, sino que busca el equilibro entre ambos ${ }^{9}$.

La dimensión «asignación de personal» presentó un importante número de percepciones negativas; un factor que comúnmente se relaciona es la complejidad del cuidado ante pacientes con más de una morbilidad. En México, sin duda, la falta de un sistema de salud basado en la prevención trae como consecuencia que no exista hospitales, número de camas y personal suficientes, en tanto el problema no se contenga desde otros ámbitos tales como la educación para prevenir o limitar los problemas de salud en la población, incluso y prioritariamente el personal de salud.

Otro aspecto que considerar es la calidad de la atención que se brinda; se infiere que a menor número de pacientes y menor complejidad se brinda mayor calidad durante la atención. Sin embargo, esta premisa no siempre se cumple, ya que en ocasiones la falta de competencias puede ser un factor determinante para omitir o retrasar parcial o totalmente el cuidado durante la jornada laboral. Otro aspecto que es importante reconocer son las restricciones o negociaciones del personal con el sindicato para evitar cubrir áreas en las cuales el ausentismo, programado o no, repercute en la planificación del recurso humano, entre otras causas de índole sectorial que afectan a la productividad y la calidad de la atención.

La dimensión «acciones del jefe para promover la seguridad» fue negativa y demanda fortalecer el liderazgo proactivo a todos niveles, basado en la confianza y el aprendizaje organizacional. El modelo de la cultura describe tres escenarios durante un EA: el primero, el error; el segundo, una acción en la que se toman riesgos; y el tercer escenario, una conducta negligente ${ }^{10}$.

Una de las estrategias para disminuir el riesgo de error desde la perspectiva del sistema es la estandarización de la información, ya que unifica intervenciones y facilita su identificación.

Por último, la percepción del personal profesional médico y de enfermería sobre el grado de SP fue de 8.35, lo cual es positivo, y se debe seguir reforzando 
mediante nuevas estrategias y políticas que impacten en la disminución de EA derivados de la atención. Estos resultados muestran una relación con las acciones que se implementan en los HRAE para madurar el sistema de gestión de calidad y, por ende, el Modelo de Seguridad del Paciente.

\section{CONCLUSIONES}

Los resultados de esta encuesta permitieron identificar el grado de cultura de SP en los HRAE, el cual se encuentra por arriba del promedio nacional en la evaluación del Cuestionario de Cultura de Seguridad del Paciente 2019 y, comparativamente con las evaluaciones realizadas en años anteriores, el comportamiento sigue siendo superior a dicho valor de referencia (8.11 en el año 2018).

Este ejercicio también motivó la elaboración del Plan de Mejora en cada HRAE, en el cual los gestores de calidad plasmaron acciones para incidir en las principales áreas de oportunidad. Algunas de estas actividades comprenden:

- Realizar la reingeniería del proceso de comunicación en el tránsito de pacientes dentro del hospital y cambios de turno.

- Elaborar el lineamiento para la entrega-recepción de guardia de área médica y del área de enfermería.

- Capacitar sobre la técnica Situación, Antecedentes, Evaluación y Recomendación (SAER) al personal operativo.

- Capacitar en EA y fomentar su reporte en el buzón institucional, ya sea electrónico o en papel.

- Capacitar a directores, subdirectores, jefes de área y de servicio en SP.

- Realizar vídeos con temas orientados a las líneas de acción para la SP.

- Capacitar en materia de SP que incluya las ocho Acciones Esenciales para la Seguridad del Paciente.

- Impartir cursos con enfoque en la promoción de valores y trabajo en equipo.
- Realizar recorridos hospitalarios de manera mensual a los servicios con participación de personal directivo.

- Realizar campañas de sensibilización en SP con apoyo de los recursos emitidos por la normatividad vigente ${ }^{11}$.

En prospectiva, la evaluación del Cuestionario de Cultura de Seguridad del Paciente 2020 permitirá observar el comportamiento de las variables con mayor área de oportunidad a fin de continuar perfeccionando los procesos.

\section{FINANCIAMIENTO}

La presente investigación no ha recibido ninguna beca específica de agencias de los sectores público, comercial o sin ánimo de lucro.

\section{CONFLICTO DE INTERESES}

Los autores declaran no tener conflicto de intereses.

\section{RESPONSABILIDADES ÉTICAS}

Protección de personas y animales: Los autores declaran que para esta investigación no se han realizado experimentos en seres humanos ni en animales.

Confidencialidad de los datos: Los autores declaran que han seguido los protocolos de su centro de trabajo sobre la publicación de datos de pacientes.

Derechos de privacidad y consentimiento informado: Los autores declaran que en este artículo no aparecen datos de pacientes.

\section{BIBLIOGRAFÍA}

1. García Pérez MÁ. Los principios de la bioética y la inserción social en la práctica médica. Rev Adm Sanit. 2006;4(2):341-56.

2. Consejo de Salubridad General. Acuerdo por el que se declara la obligatoriedad de la implementación, para todos los integrantes del Sistema Nacional de Salud, del documento denominado Acciones Esenciales para la Seguridad del Paciente [Internet]. México: Diario Oficial de la Federación; 08/09/2017. Disponible en: www.dof.gob.mx/nota_detalle.php?codigo=549 $6728 \&$ fecha $=08 / 09 / 2017$

3. Aranaz-Andrés JM, Aibar-Remón C, Sarabia 0. Diseño del estudio IBEAS: Prevalencia de efectos adversos en hospitales de Latinoamérica. Rev Calid Asist. 2011;26(3):194-200. 
4. Secretaría de Salud. Dirección General de Calidad y Educación en Salud. Instrucción 337/2012: Evaluación de la Cultura de Seguridad del Paciente en Hospitales de México [Internet]. México: Secretaría de Salud. Dirección General de Calidad y Educación en Salud; 2012. Disponible en: http://www. calidad.salud.gob.mx/site/calidad/instruccion/instruccion_337.pdf

5. Sarabia 0. Acciones esenciales en seguridad del paciente. Marzo 2018 [Internet]. Gobierno de México [consultado: 15 de enero de 2019]. Disponible en: http://www.calidad.salud.gob.mx/site/calidad/docs/acciones_ esenciales.pdf

6. Encuesta. Cultura de seguridad del paciente. Resultados 2019 [Internet]. México: Secretaría de Salud, Subsecretaría de Integración y Desarrollo del Sector Salud, Dirección General de Calidad y Educación en Salud; 2019. Disponible en: http://dgces.salud.gob.mx/encuesta/culturasp/resultados. php

7. La alianza mundial por la seguridad del paciente. Marco conceptual de la clasificación internacional para la seguridad del paciente. Versión 1.1. Informe técnico definitivo [Internet]. Organización Mundial de la Salud; en- ero de 2009 [consultado: 15 de enero de 2019]. Disponible en: http://www. who.int/patientsafety/implementation/icps/icps_full_report_es.pdf

8. Aranaz J, Aibar C, Vitaller J, Ruiz P. Estudio nacional sobre los efectos adversos ligados a la hospitalización. ENEAS 2005. Madrid: Ministerio de Sanidad; 2006.

9. Rocco C, Garrido A. Seguridad del paciente y cultura de seguridad. Rev Med Clin Las Condes. 2017;25(5):785-95.

10. Pérez J. Seguridad del paciente al alcance de todos [Internet]. México: Centro Virtual de Operaciones en Emergencias y Desastres (CVOED); septiembre de 2013 [consultado: 15 de enero de 2019]. Disponible en: http:// cvoed imss.gob. $\mathrm{mx} / \mathrm{COED} / \mathrm{home} /$ normativos/DPM/archivos/coleccionmedicinadeexcelencia/20\%20Seguridad\%20del\%20paciente $\% 20$ al $\% 20$ alcance $\% 20$ de $\% 20$ todos-Interiores.pdf

11. Plan de Mejora de los Hospitales Regionales de Alta Especialidad, octubre 2019. México: Secretaría de Salud, Comisión Coordinadora de institutos Nacionales de Salud y Hospitales de Alta Especialidad, Dirección General de Coordinación de los Hospitales Regionales de Alta Especialidad; 2019. 